LBL-33982

UC-414

\title{
NEW a-Si:H PHOTO-DETECTORS \\ FOR LONG-TERM CHARGE STORAGE
}

H. Lee, G. Cho, J.S. Drewery, W.S. Hong, T. Jing, S.N. Kaplan, A. Mireshghi, V. Perez-Mendez and D. Wildermuth

Physics Division

Lawrence Berkeley Laboratory

University of California

Berkeley, CA 94720

APRIL 1993

This work was supported by the Director, Office of Energy Research, Office of Basic Energy Sciences, Division of High Energy Physics, of the U.S. Department of Energy under Contract No. DE-AC03-76SF00098. 


\title{
NEW a-Si:H PHOTO-DETECTORS \\ FOR LONG-TERM CHARGE STORAGE
}

H. LEE, G. CHO, J. S. DREWERY, W. S. HONG, T. JING, S. N. KAPLAN, A. MIRESHGHI, V. PEREZ-MENDEZ AND D. WILDERMUTH

Lawrence Berkeley Laboratory, Berkeley, CA 94720

\begin{abstract}
Using the high light absorption properties of amorphous silicon, we developed a new device configuration that can detect photons and store the induced charges for relatively long time. This device, coupled to a scintillator such as CsI(TI) in an array form, could be used as a scintillation camera, or for long-term photo-detection such as radionuclide labeled chromatography. The detector has a simple sandwich structure consisting of a scintillator followed by a top metal layer, $p-i-n$ layers of hydrogenated amorphous silicon (a-Si:H), a second metal layer, a thin insulating layer and a bottom metal layer. The electron-hole pairs generated in the i-layer by the interaction with the incident light will be separated by the imposed electric field and be stored in the central metal-insulator interface. Readout will be done by switching the external bias to ground after the storage time, which depends on the needs for the specific application. Prototype devices were fabricated and tested. The performances of the devices were analyzed in connection with the storage time and the background signal produced by the thermally generated charges.
\end{abstract}

\section{INTRODUCTION}

Hydrogenated amorphous silicon (a-Si:H) devices have been described for radiation detection applications.[1] Due to the large area and high radiation resistivity, a-Si:H radiation detectors have some advantages over the crystalline devices. There are many applications of a-Si:H radiation detectors which include detection of visible light, $x$-rays, $\gamma$-rays, charged particles and neutrons.[1-4] For example, for the imaging of $\gamma$-ray distribution in nuclear medicine we can replace a conventional scintillation camera with an a-Si:H pixel detector combined with a scintillator. CsI(TI) is known to be an appropriate scintillator for this purpose because of its high light yield and high interaction efficiency for $\gamma$ rays.[4] While the intrinsic spatial resolution of conventional scintillation cameras is more than $3 \mathrm{~mm}$, a-Si:H pixel devices can be made to have much higher spatial resolution if required. Moreover, these devices do not utilize photomultiplier tubes (PMTs) unlike the conventional cameras and accordingly have the advantage of compactness and light weight. With ordinary $\mathrm{p}-\mathrm{i}-\mathrm{n}$ a-Si:H pixel detectors for this purpose we would have to record each incoming $\gamma$ ray by low noise multistage amplifiers at each pixel because of the low counting rate. Storage and integration of the individual counts to give a larger signal can eliminate the need for pixel level amplifiers thus making the readout scheme much simpler.

In this paper we describe a device which is appropriate for this purpose. This consists of a scintillator placed on the top transparent conducting layer followed by $p$ $\mathrm{i}-\mathrm{n}$ a-Si:H layers, a second metal layer, an insulating layer and a bottom metal layer as shown in Fig. 1. By applying a reverse bias the i-region is under an electric field. When the light is incident on it, it produces electron-hole pairs and these carriers will 


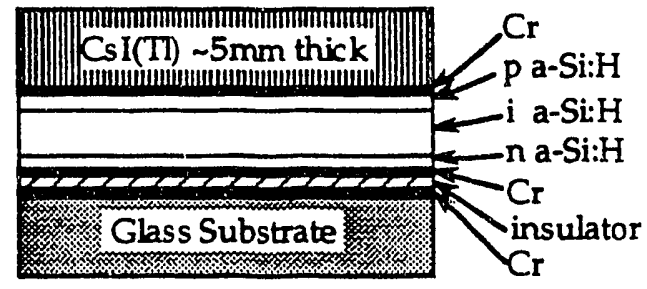

Fig. 1 A schematic diagram of a charge storing detector. Top $\mathrm{Cr}$ is made semitransparent for light penetration. Silicon dioxide or silicon nitride was used for the insulator.

drift to the top and middle metallic layers and remain there until the electric field is reversed. In order to read out the amount of stored charges the applied bias is switched to ground and by integration of the measured current we can obtain the charge information.

\section{EXPERIMENTS}

Oxide $\left(\mathrm{SiO}_{2}\right)$ or nitride $\left(\mathrm{Si}_{3} \mathrm{~N}_{4}\right)$ were used as the insulators and the thicknesses were 200 300 nm. Thin insulators are preferred in order to make a high capacitance of the metal-insulator-metal which can accommodate a large amount of charge. The $\mathrm{p}$ - and n-layer of a-Si:H were $30 \mathrm{~nm}$ and $20 \mathrm{~nm}$ thick respectively and the i-layer was $1 \mu \mathrm{m}$ thick which was calculated to absorb 96\% of the light emitted from the CsI(TI) using the emission spectrum of $\mathrm{CsI}(\mathrm{TI})[4]$ and optical absorption coefficient of a-Si:H.[5] The capacitance of the insulator, $C_{i}$, was $1700 \sim 3300 \mathrm{pF}$ and that of the $\mathrm{p}-\mathrm{i}-\mathrm{n}$ diode, $\mathrm{C}_{\mathrm{d}}$, was $-1150 \mathrm{pF}$ for a pixel size of $3 \mathrm{~mm} \times 3 \mathrm{~mm}$.

The experimental system and the pulse configuration from each pulse generator are shown in Fig. 2. The p-i-n diode in the device is indicated as a capacitor $\mathbf{C}_{\mathbf{d}}$ with a resistor $\mathbf{R}_{\mathbf{d}}$ in parallel and the metal-insulator-metal layer is indicated as a capacitor $C_{i}$. In the system configuration there is another capacitor $C_{c}$ and a resistor $R_{c}$, which are used to cancel out the RC transients when the bias from PG1 changes. The capacitance of $C_{c}$ is equal to the total capacitance of $C_{d}$ and $C_{i}$ in series and the resistance of $R_{c}$ is equal to the forward resistance of the $p-i-n$ diode which is $\sim 100 \mathrm{k} \Omega$. By applying pulses of the same magnitude but opposite polarities on the charge

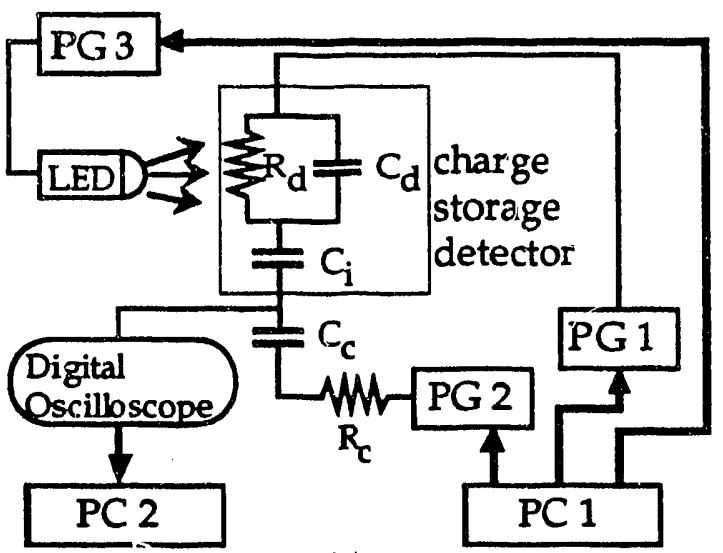

(a)

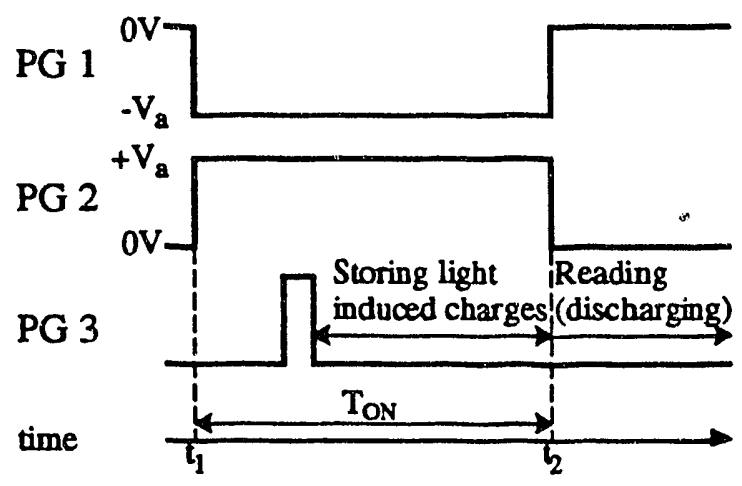

(b)

Fig. 2 (a) Schematic representation of the experimental system. IBM PC 1 controls the pulse generators and PC 2 is used for data acquisition. The p-i-n a-Si:H diode is indicated as a resistor $R_{d}$ and a capacitor $C_{d}$ in parallel.

(b) The time sequence of the pulses from the pulse generators. At $t_{1}$ the charge storing detector begins to store the charges generated by either incident light or thermal excitation. 
storage detector and on the $R_{C}-C_{c}$ components simultaneously the switching transients were canceled up to $98 \%$. The residual transients were measured separately and subtracted from the signal. The time sequence of the operation is shown in Fig. 2 (b). During $\mathrm{T}_{\mathrm{ON}}$ a reverse bias, $\mathrm{V}_{\mathrm{a}}$ is applied on the device. The light incident during this interval generates electron-hole pairs, which will be stored until time $t_{2}$. Then the stored charges are discharged by switching the bias to $0 \mathrm{~V}$. The discharging current is read by the digital oscilloscope and the amount of the stored charges is obtained by integration of the current.

\section{RESULTS}

When a step pulse of the reverse bias, $V_{a}$ is applied on the device, the $p-i-n$ diode acts like a capacitor and charges build up on its surfaces as well as on the insulator surfaces in a few system $R C$ times, then the voltage across the $p-i-n$ diode, $V_{d}$, and across the insulator, $V_{i}$, is set by the corresponding capacitance ratio, i. e., $V_{d}=$ $\mathrm{V}_{\mathrm{a}} \mathrm{C}_{\mathrm{i}} /\left(\mathrm{C}_{\mathrm{d}}+\mathrm{C}_{\mathrm{i}}\right)$ and $\mathrm{V}_{\mathrm{i}}=\mathrm{V}_{\mathrm{a}} \mathrm{C}_{\mathrm{d}} /\left(\mathrm{C}_{\mathrm{d}}+\mathrm{C}_{\mathrm{i}}\right)$. Due to the reverse bias $\mathrm{V}_{\mathrm{d}}$, thermally generated carriers in the i-region flow along the electric field and are stored on the insulator surfaces. As the thermal generation current flows, charges will accumulate on the insulator capacitor and correspondingly $V_{d}$ will decrease. This thermal generation current will flow until $V_{i}=V_{a}$, when the insulator capacitor is saturated with charges and the amount is given by $Q_{s a t}=V_{a} C_{i}{ }^{2} /\left(C_{d}+C_{i}\right)$. To measure the light induced charges we should read them out before the electric field in the $\mathrm{p}-\mathrm{i}-\mathrm{n}$ diode drops below full depletion, then these charges are obtained by subtracting the thermally generated charges from the total collected charges. If the thermal generation current is too large the insulator capacitor will be saturated with charges in a short time and the light induced charges cannot be stored with full efficiency.

One way to store the light induced charges for a long time is to have a high value of $Q_{\text {sat }}$ by applying high $V_{a}$ and making $C_{i}$ high and $C_{d}$ low. The values of $C_{i}$ and $C_{d}$ can be manipulated by adjusting the areas and the thicknesses of the corresponding layers. Another way of having a long storage time is to reduce the thermal generation current. This can be accomplished by lowering the ambient temperature. Fig. 3 shows the temperature effects on the thermal generation current and correspondingly stored charges. At room temperaiure, $10 \mathrm{~min}$. after $10 \mathrm{~V}$ of $\mathrm{V}_{\mathrm{a}}$ is applied the thermal generation charges are accumulated almost up to the saturation level and there is

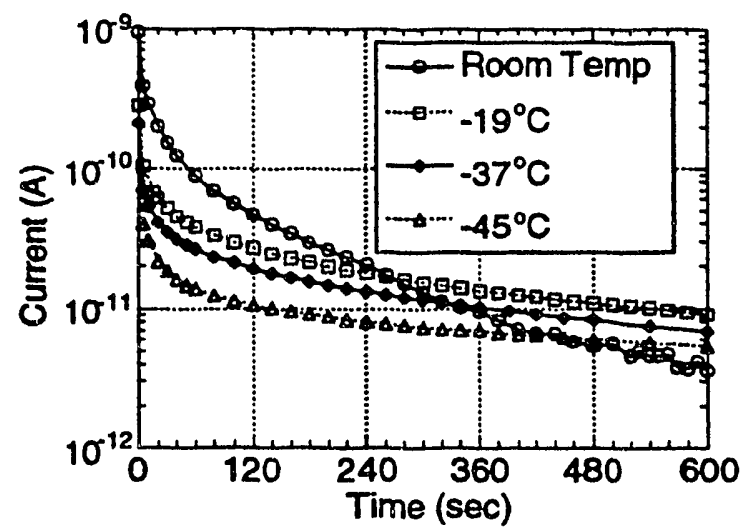

(a)

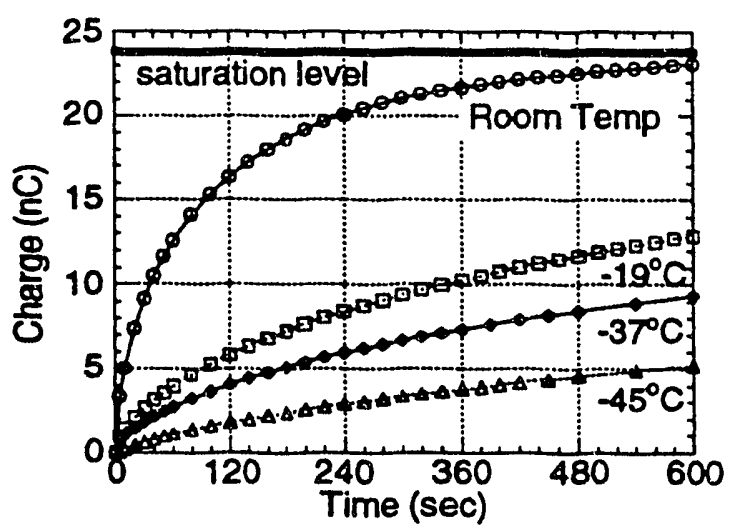

(b)

Fig. 3 Time dependent thermal generation current (a), and stored thermal generation charges (b) after applying $10 \mathrm{~V}$ on the charge storing detector at various temperatures. $\left(C_{i}=3200 \mathrm{pF}\right.$ and $\left.C_{d}=1150 \mathrm{pF}\right)$. 


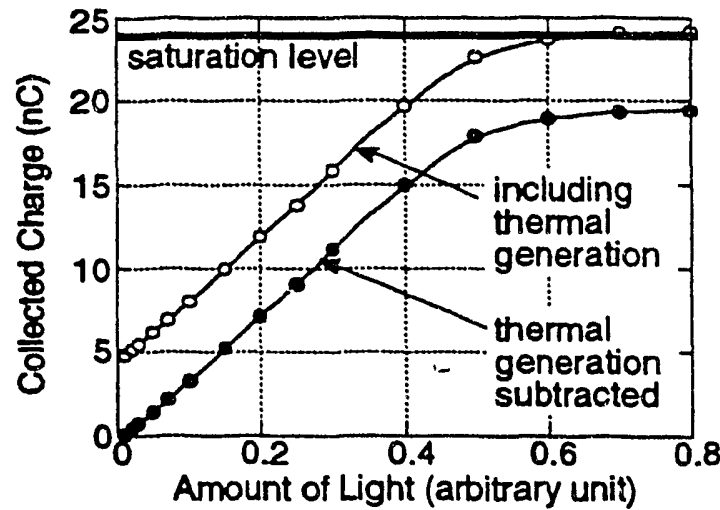

Fig.4 Relationship between the input light amount and the collected charges after 5 $\min$ storage time with a bias of $10 \mathrm{~V}$ at $\mathrm{T}=$ $-37^{\circ} \mathrm{C}$.

little room for the light induced charges. At $\mathrm{T}=-45^{\circ} \mathrm{C}$, however, the amount of the stored thermal generation charge after $10 \mathrm{~min}$. is about $21 \%$ of the $Q_{\text {sat }}$ and it takes as long as 9 hours to reach the saturation level.

As a test light source an LED of $\lambda=665 \mathrm{~nm}$ was used. With a pulse width of $100 \mathrm{~ms}$ it produced about $3 \mathrm{nC}$ of charges in the i-region. A relationship between the input light amount and the collected charges after $5 \mathrm{~min}$. of storage time at $\mathrm{T}=-37^{\circ} \mathrm{C}$ is shown in Fig. 4. We can see that there is a linear relationship between the input and the output up to $\sim 80 \%$ of saturation charge amount.

\section{DISCUSSION}

It is generally known that the thermal generation current is due to the carriers which are excited from deep states to the band edges and drift along the depletion field. Theoretical models of the thermal generation current have been developed using a single trap level and a Shockley-Read-Hall formalism.[6,7] The thermal generation current is also significantly dependent on voltage and this is due to the field enhanced emission such as Poole-Frenkel effect, tunneling and phonon assisted tunneling. [8-10] In the range of bias and temperature with which our experiments were performed, the Poole-Frenkel effect was found to be the dominant one, and the other effects may be neglected.

The thermal generation current with Poole-Frenkel effect only can be expressed as

$$
I_{R}=\alpha(k T)^{4} \frac{\sqrt{F}}{\beta_{P F}} \exp \left[\frac{-E_{i}+\beta_{p_{F}} \sqrt{F}}{k T}\right]
$$

where $\alpha$ is a parameter which depends on the geometry and material properties of the device, $F$ is the electric field, $\beta_{P F}$ is the Poole-Frenkel constant, $E_{i}$ is the difference between conduction band mobility edge and quasi-Fermi energy.

By finding a time dependence of the electric field $F$ in Eq. (1) we can obtain the time dependence of the thermal generation current also. By approximation the $p-i-n$ diode in Fig. 2 (a) may be considered as a voltage dependent current source with a capacitor, $C_{d}$, in parallel. Then by a circuit analysis with Eq. (1) as the current source expression we can get the time dependence of $F$ which is assumed to be uniform, i. e., $F=V_{d} / d$. The resulting electric field is expressed as 


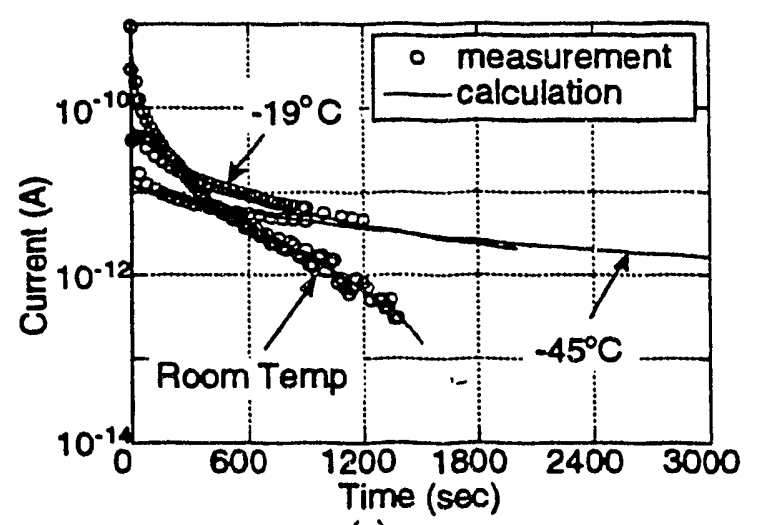

(a)

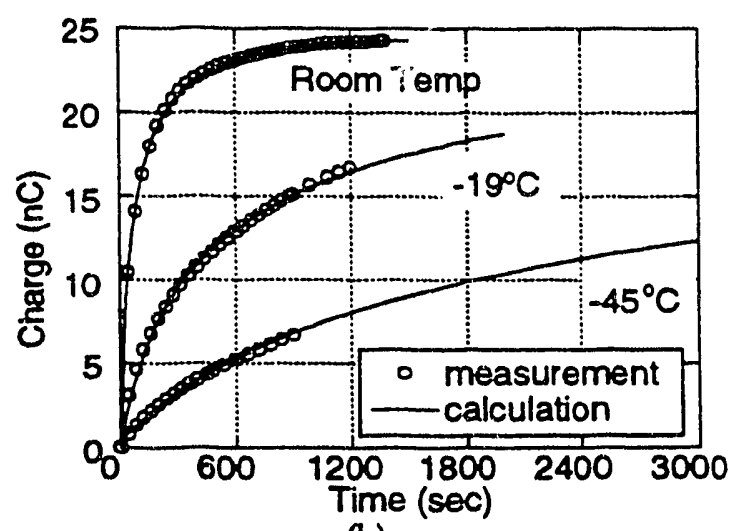

(b)

Fig. 5 Comparisons of measurements and theoretical calculations for the time dependent thermal generation current (a), and stored charges (b) at various temperatures. $\alpha=0.15, \beta_{\mathrm{PF}}=0.04$ and $E_{i}=0.34$ were used in Eq. (1) and (2) for the fittings.

$$
F(t)^{1 / 2}=\frac{k T}{\beta_{P F}} \ln \left[\exp \left(-\frac{\beta_{P F}}{k T} \sqrt{\frac{C_{i} V_{a}}{\left(C_{i}+C_{d}\right) d}}\right)+\frac{\alpha t}{2 C_{i} d}(k T)^{3} \exp \left(-\frac{E_{i}}{k T}\right)\right]
$$

where $d$ is the thickness of the i-layer.

By assigning proper values to the parameters $\alpha, \beta_{P F}$ and $E_{i}$ in Eq. (1) and (2) good fits to the measurements were obtained. For the best fit $\alpha=0.15 \mathrm{Amp}-\mu \mathrm{m} / \mathrm{V} \cdot \mathrm{eV}^{3}, \beta_{p F}=$ $0.04 \mathrm{eV} \sqrt{\mu \mathrm{m} / \mathrm{V}}$ and $E_{i}=0.34 \mathrm{eV}$ were given and the comparisons of the calculated thermal generation currents and the stored charges and those obtained from measurements are shown in Fig. 5. The value of $\beta_{P F}$ is about twice larger than the theoretical value of 0.022 . This increase may be interpreted that the Poole-Frenkel effect is enhanced because the surrounding defect states give screening effect resulting in lowering of the barrier height further.[10] $E_{i}$ has been measured by many workers and is $0.9 \sim 0.95 \mathrm{eV}$ at steady state and $0.75 \sim 0.8 \mathrm{eV}$ at initial transient of applying reverse bias. [6,7] The value of $E_{i}$ for our fitting was $0.41 \sim 0.61 \mathrm{eV}$ lower than the conventional values. This may be due to significant emissions from the shallow states.

The time to reach $90 \%$ of the saturation level by thermal generation current, using the above model with $\mathrm{V}_{\mathrm{a}}=10 \mathrm{~V}, \mathrm{C}_{\mathrm{d}}=1150 \mathrm{pF}$ and $\mathrm{C}_{\mathrm{i}}=3200 \mathrm{pF}$, is plotted as a function of temperature in Fig. 5. As the temperature decreases the saturatior time increases exponentially with the activation energy of $0.38 \mathrm{eV}$ and at $\mathrm{T}=-50^{\circ} \mathrm{C}$ the saturation time is about 13 hours. As the saturation time is so sensitive to the temperature, the

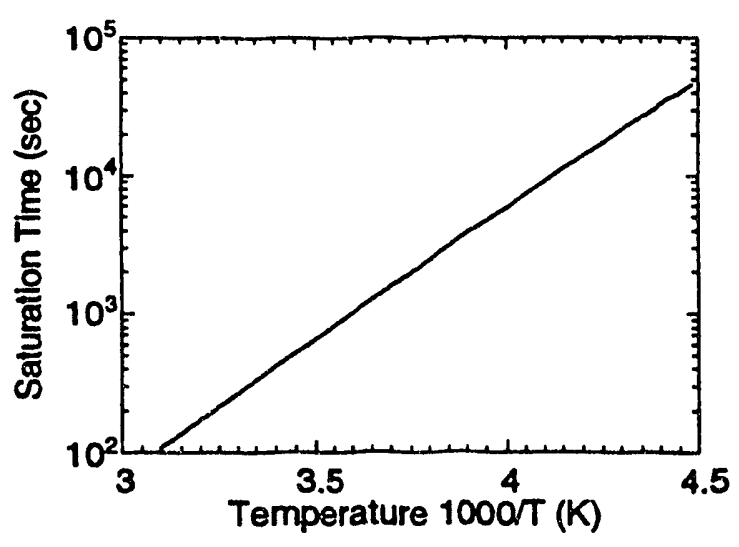

Fig.6 Temperature dependence of the saturation time which is defined as the time needed to store the thermal generation charges up to $90 \%$ of $Q_{\text {sat. }}$. 
maximum storage time can be easily manipulated by controlling the temperature.

\section{CONCLUSION}

We have given a discussion of our studies of a detector which is able to store the light induced charges for a long time. With our prototype devices at room temperature, significant thermal generation current flows and saturates the insulator capacitor in $10 \mathrm{~min}$. witt $10 \mathrm{~V}$ of applied bias. The thermal generation current, however, could be lowered by reducing the ambient temperature, and after $10 \mathrm{~min}$. at $\mathrm{T}=-45^{\circ} \mathrm{C}$ the stored thermal generation charges were about $21 \%$ of the saturation charge amount. Another way to reduce the thermal generation current is to make the volume of $\mathrm{p}-\mathrm{i}-\mathrm{n}$ diode smaller by reducing the contact area, but the thickness should be thick enough to ensure low capacitance of $p-i-n$ diode because lower capacitance of the $p-i-n$ diode require a higher initial bias on it and a higher $Q_{\text {sat. }}$. By the same reasons high capacitance of the insulator, i. e., thin insulator, is preferred. A high value of the $\mathrm{V}_{\mathrm{a}}$ also provides a long storage time. A good linear relationship between the input light and the output charges was acquired up to $\sim 80 \%$ of $Q_{\text {sat }}$ with a storage time of $5 \mathrm{~min}$.

A time dependent thermal generation current model based on the previous models was developed for our experiments and it gave good fits to the measurements. Using this model the amount of stored thermal generation charges and the maximum storage time at any temperature can be predicted. The time needed to saturate the insulator capacitor with thermal generation charges has an exponential dependence on the reciprocal of the temperature and with slight decrease in temperature much longer storage time can be obtained.

\section{ACKNOWLEDGEMENTS}

This work was supported by the US Department of Energy under contract No. DE-AC03-76SF00098.

\section{REFERENCES}

1. V. Perez-Mendez, J. Morel, S. N. Kaplan and R. A. Street, Nucl. Instr. and Meth. A252, 478 (1987).

2. V. Perez-Mendez, in Amorphous \& Microctystalline Semiconductor Devices, edited by J. Kanicki (Artech House, Boston, 1991), pp. 297-330.

3. A. Mireshghi, G. Cho, J. Drewery, T. Jing, S. N. Kaplan, V. Perez-Mendez and D. Wildermuth, IEEE Trans. Nuc. Sci. NS-39, 635 (1992).

4. I. Fujieda, G. Cho, J. Drewerey, T. Gee, T. Jing, S. N. Kaplan, V. Perez-Mendez and D. Wildermuth, IEEE Trans. Nuc. Sci. NS-38, 255 (1991).

5. G. D. Cody, in Semiconductors and Semimetals, Vol. 21, Part B volume edited by J. I. Pankove (Academi Press, Orlando, 1984), p. 23.

6. R. A. Street, Appl. Phys. Lett. 57 (13), 1334 (1990).

7. R. A. Street, Philos. Mag. 63 (6), 1343 (1991).

8. E. Rosencher, V. Mosser and G. Vincent, Phys. Rev. B 29 (3), 1135 (1984).

9. G. Vincent, A. Chantre and D. Bois, J. Appl. Phys. 50 (8), 5484 (1979).

10. R. M. Hill, Philos. Mag. 23, 59 (1971). 


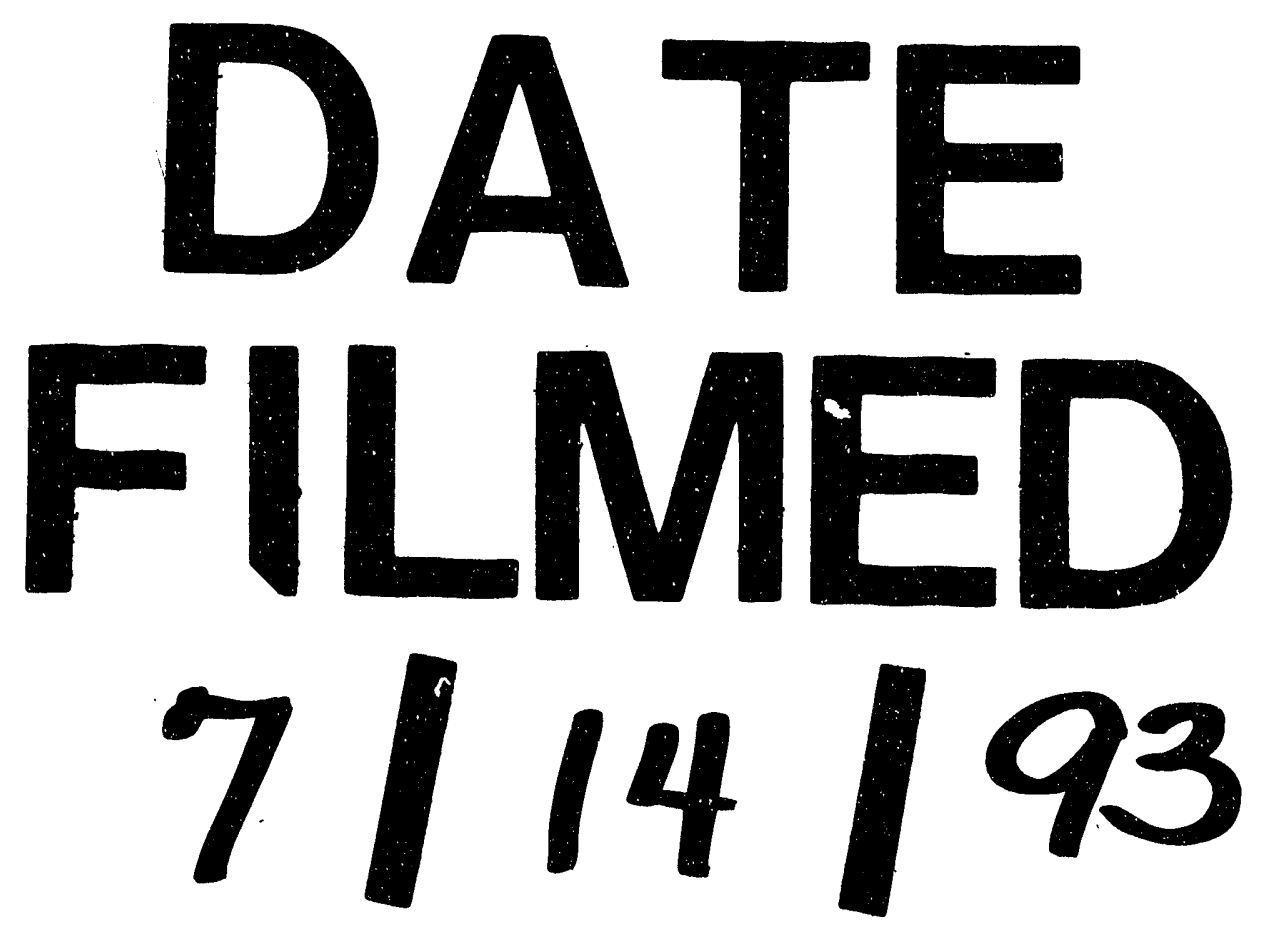


3

$\|$ 